\title{
Consideraciones teóricas y metodológicas para investigar sobre deserción escolar
}

\author{
Román Hernández -Dávila ${ }^{a}$, Waditt Enrique Díaz- Abdala ${ }^{\mathrm{b} *}$ \\ aDoctor en pedagogía, Universidad Francisco de Paula Santander; \\ ${ }^{\text {b} E s p e c i a l i s t a ~ e n ~ d e r e c h o s ~ h u m a n o s, ~ U n i v e r s i d a d ~ d e ~ S a n t a n d e r, ~ U D E S . ~ C u ́ c u t a . ~}$ \\ Forma de citar: Hernández, R. \& Diaz, W. (2017). Consideraciones teóricas y metodológicas para \\ investigar sobre deserción escolar. Perspectivas, 2(2). 108-119.
}

Recibido: enero 03 de 2017

Aceptado: abril 25 de 2017

\begin{abstract}
Palabras clave
Contextos sociales vulnerables, deserción escolar, gestión escolar, metodología cualitativa
\end{abstract}

Resumen: Este trabajo ofrece un exhaustivo análisis de diferentes autores y experiencias de investigación relacionadas con criterios generales y metodológicos sobre los motivos que generan la deserción escolar, principalmente en ámbitos vulnerables, para lo cual es necesario reflexionar sobre el problema con la intención de describir las variables que lo ocasionan. En estas situaciones inciden diversos elementos que abarcan desde la organización de los sistemas educativos, la aplicación de políticas públicas, las condiciones del contexto social, la situación familiar e individual, la salud, los efectos psicológicos y afectivos involucrados y las pautas culturales que envuelven a las familias de los menores, hasta la gestión escolar y la relación de los estudiantes con los docentes. Como aporte de esta revisión se ofrece una serie de propuestas útiles en la generación de cambios en las dinámicas educativas enfocadas en la superación de la deserción escolar. Se asume que la investigación sobre deserción se debe emprender desde una concepción cualitativa, con características hermenéuticas, que permitan interpretar con fehaciencia el fenómeno a estudiar. Esta base metodológica tiene fundamentos

\footnotetext{
*Autor para correspondencia wa.diaz@mail.udes.edu.co
} 
etnográficos, pues requiere revisar aspectos específicos propios de contexto sociocultural presentes en las variables sujetas a investigación. Los trabajos de esta naturaleza deben explicar los factores determinantes de la deserción escolar en un ámbito específico de estudio, describirlos y generar una base de datos e información que visualice los indicadores de las causas generadoras del fenómeno.

\section{Keywords}

Vulnerable social contexts, school dropout, school management, qualitative methodology

\section{Palavras chave}

Contextos sociais vulneráveis, abandono escolar, gestão escolar, metodologia qualitativa

\section{Theoretical and methodological considerations to investigate school dropout}

\begin{abstract}
This work offers an exhaustive analysis of different authors and studies- falling under general and methodological criteria - of the reasons responsible for school dropouts, predominantly in vulnerable areas. To do so it is necessary to reflect on the problem with the intention of identifying the contributing factors. In these situations, various elements come into play, ranging from the organization of education systems, application of public policies, social conditions, the individual's situation, health, the psychological and emotional impact, as well as the cultural patterns that may in compass the minor's family, student-teacher relationship and school management. As an addition, a series of useful proposals is offered in bringing about change in the educational sector, focused on the reducing of school dropouts. It is assumed that research about dropouts should be undertaken from a qualitative concept, with hermeneutical characteristics, that allow the phenomenon under study to be interpreted reliably. This methodological basis has ethnographic foundations since it requires the reviewing of specific aspects of the socio cultural context present in the factors subject to investigation. Works of this nature should identify the determinants of school dropouts in a specific line of inquiry, describe them, and generate a database that displays the indicators of the causes that create this phenomenon.
\end{abstract}

\section{Considerações teóricas e metodológicas para investigar o abandono escolar}

Resumo: Este trabalho oferece uma análise exaustiva de diferentes autores e experiências de pesquisa relacionadas a critérios gerais e metodológicos sobre os motivos que levam ao abandono escolar, principalmente em áreas vulneráveis, para as quais é necessário refletir sobre o problema com a intenção de descrever as variáveis que eles causam isso. Nessas situações, vários elementos que vão desde a organização dos sistemas educacionais, a aplicação das políticas públicas, as condições do contexto social, a situação familiar e 
individual, a saúde, os efeitos psicológicos e afetivos envolvidos e os padrões culturais que cercam as famílias dos menores, até a gestão da escola e a relação dos alunos com os professores. Como contribuição desta revisão, uma série de propostas úteis são oferecidas na geração de mudanças nas dinâmicas educacionais voltadas para a superação de abandono escolar. Supõe-se que a pesquisa sobre deserção deve ser realizada a partir de uma concepção qualitativa, com características hermenêuticas, que permitam que o fenômeno seja estudado para ser interpretado com fé. Esta base metodológica tem fundamentos etnográficos, uma vez que requer a revisão de aspectos específicos do contexto sociocultural presente nas variáveis sujeitas à pesquisa. Os trabalhos desta natureza devem explicar os determinantes do abandono escolar em um campo de estudo específico, descrevê-los e gerar um banco de dados e informações que visualizam os indicadores das causas que geram o fenômeno.

\section{Referentes generales de organismos internacionales sobre la deserción escolar}

La deserción o abandono escolar es un fenómeno cuyo incremento en la actualidad hace indispensable su abordaje en el marco más general del estudio de la mejora de la calidad educativa. Organismos como la UNESCO dan prioridad a la inclusión y el mantenimiento de los estudiantes en el sistema en cuanto soporte de las expectativas educativas, para lo cual es necesario controlar los factores que interrumpen la prosecución en la educación, sobre todo en los niveles de educación básica y media.

Con frecuencia inciden en este fenómeno los problemas económicos, familiares, el escaso nivelde estudio de los padres, los ambientes socioculturales que rodean las comunidades deprimidas donde están ubicados los estudiantes, la violencia intrafamiliar, la violencia en las escuelas o en los entornos vulnerables, así como los factores denominados por algunos especialistas e instituciones (entre ellos, el National DropoutPrevention Center, 2017) como A, B, C, es decir, ausentismo, conductas desviadas y rendimiento (attendance, behavior, course, performance, por sus siglas en inglés).
Según los datos de la CEPAL, para el año 2009 menos de la mitad de los jóvenes consiguieron terminar el nivel educativo medio en Latinoamérica, lo cual está en relación, además de los factores mencionados, con la relación de los alumnos con los docentes, el universo simbólico de las familias y las escuelas en el desarrollo de expectativas.

Vista así, la deserción es un proceso multifactorial que posee características particulares de acuerdo con los contextos de estudio. En este enfoque se revisan las peculiares causas que la originan con la intención de apreciar los factores citados y las carencias pedagógicas intervinientes.

Para superar el fenómeno de la deserción escolar se debe ofrecer servicios de calidad, sean del Estado, de las instituciones públicas y privadas o de alianzas que impliquen estrategias para resolver el abandono escolar. Desde el universo de las prácticas pedagógicas deben surgir también propuestas didácticas que motiven al estudiante a involucrarse en actividades que favorezcan los estados emocionales para hacerlos más sociables.

En ese escenario, se pretende describir con sentido crítico las causas que generan deserción y proponer estrategias que permitan detectar a tiempo los posibles casos para atenderlos de manera individualizada y sistemática, y así establecer 
propuestas pedagógicas y atención integral social que mitiguen el abandono de la escuela y estimulen su continuidad en ella.

El fenómeno de la deserción escolar es considerado crucial de superar para llegar a una educación más inclusiva, participativa y democrática que influya determinadamente en el crecimiento económico con equidad.

Pese a los acuerdos y proyecciones de la comunidad internacional para alcanzar una educación para todos en el año 2015, en el informe de la UNICEF y el Instituto de Estadística de la UNESCO (2015) se señala que "63.000.000 de adolescentes entre 12 y 15 años están privados del derecho a la educación".

Estas cifras incluyen tanto a quienes nunca han asistido a la escuela como a los desertores o decepcionados. Ello, a pesar de que en muchos países latinoamericanos los estudios sean prácticamente gratuitos" (Esteban, 2016: 5).

Actualmente se insiste mucho en que la inclusión y la equidad deben ser metas prioritarias en las políticas públicas para alcanzar índices de crecimiento sustentable, que tengan como fundamento el desarrollo de las fuerzas productivas. Mientras más estudiantes estén incorporados a los procesos educativos, en particular en los niveles de básica y media, más posibilidades habrá de mejora en las condiciones de vida social y personal; y la evidencia, de acuerdo con Esteban (2016: 5), indica que terminar la educación secundaria se está volviendo más necesario para evitar situaciones de marginalidad laboral y social en la región.El nuevo estándar para evaluar los progresos debiera ser qué proporción de las generaciones más jóvenes completan efectivamente este ciclo de enseñanza.

Por ello es preciso incidir desde el Estado en propiciar mejores condiciones de vida para los sectores más desprotegidos social $\mathrm{y}$ económicamente. Sobre el particular, Abril Valdez, Román Pérez, Cubillas Rodríguez y Moreno Celaya (2008: 1) señalan que los resultados muestran la necesidad de un modelo de intervención basado en políticas educativas con mayores incentivos para una adherencia al sistema escolar, flexibilización del tránsito entre sub sistemas y reestructuración de las redes de comunicación entre los actores principales.

Todas las evidencias indican que los principales factores asociados a la deserción se conjugan en las carencias socioculturales y económicas existentes en los sectores vulnerables. Las condiciones de pobreza y pobreza extrema, el desempleo, la deplorable situación de salud que conlleva a la desnutrición, la violencia instalada, intrafamiliar o delincuencial, presente en los barrios marginales, el escaso nivel educativo de padres y madres, las constantes migraciones, el mundo del consumo y el tráfico de drogas, obligan a grandes masas de infantes y jóvenes a abandonar las precarias escuelas instaladas en zonas urbanas y rurales.

En América Latina la situación de la deserción escolar ha impactado de manera negativa las posibilidades de desarrollo en la región. No se han cumplido las metas de inclusión, que supondrían que en los niveles de básica y secundaria los alumnos se mantengan y avancen y puedan ser incorporados con un mínimo de calidad productiva a las fuentes de empleo y de trabajo digno.

Sobre el particular, la UNESCO (2013: 8788), diagnostica que dado que la evidencia indica que terminar la educación secundaria se está volviendo más necesario para evitar situaciones de marginalidad laboral y social en la región, el nuevo estándar para evaluar los procesos debiera ser qué proporción de las generaciones más jóvenes completa efectivamente este ciclo de enseñanza.

La preocupación sobre el tema está encaminada, por un lado, a atacar la pobreza que afecta a las comunidades de las que provienen los alumnos, pues la ignorancia y el desempleo en ocasiones conducen a la incorporación de los niños en edad escolar a fuentes de ingreso provenientes de actividades ilícitas. Por otro, la buhonería, el trabajo informal y la explotación infantil facilitan su vinculación temprana con el delito y el abandono de la escuela.

Spindola y León (2002) incorporan un importante criterio en la vinculación que debe existir 
entre años de escolaridad y saberes necesarios para mantenerse en las esferas del trabajo productivo, en particular cuando los alumnos desertan antes de concluir la educación media. Al respecto aseguran que no alcanzan "el capital educacional mínimo y las destrezas requeridas para mantenerse fuera de la pobreza durante la vida activa, incumpliéndose así los derechos a la educación consagrados en las declaraciones internacionales pertinentes (2002: 3)."

Esos hechos repercuten tanto en la calidad de vida de los afectados como en la baja producción y productividad en el trabajo, menoscabando además, derechos como los de la salud, trabajo digno, la equidad y la protección del menor.

El Programa de las Naciones Unidas para el Desarrollo (PNUD) ha insistido desde la década de los noventa en la necesidad de relegar el concepto de desarrollo basado en la generación de riqueza por vía exclusiva del aumento del PIB, el índice per cápita, la explotación de materias primas y la utilización de mano de obra barata. En su lugar, este organismo propone humanizar el desarrollo de los países mediante mayor inversión en salud, educación y protección de los derechos fundamentales como base para lograr modos de vida sustentables y de mayor progreso.

Estos tres aspectos fundamentales en las concepciones del PNUD sobre el desarrollo están relacionados con los factores sociales que inciden con mayor potencia en el desapego a la escuela y la consecuente deserción escolar. Construir espacios de logros en el avance escolar de los estudiantes constituye un signo de encuentro, inclusión y desarrollo económico con justicia social y equidad.

Como se deduce de lo anterior, una concepción de esta naturaleza potenciaría más las inversiones del Estado para crear una ciudadanía más participativa y responsable, capaz de mejorar las fuerzas productivas con talento que impacte cualitativamente sobre las condiciones de trabajo y las transformaciones que requieren los productos y servicios en beneficio de las mayorías nacionales. Para ello se hace necesario procurar por todos los medios posibles que los alumnos se mantengan en las escuelas y nutrir de calidad su educación, lo cual impactaría positivamente sobre la concepción del desarrollo.

Otro aspecto vinculado con la deserción escolar es cómo hacer posible que los docentes en los niveles iniciales sepan atender, desde las perceptivas pedagógica y didáctica, los problemas que surgen en el aula como producto de la conflictividad social, la salud, los divorcios, la promiscuidad, la pobreza crítica, la violencia intrafamiliar y las diferencias etarias, todo lo cual provocan en los estudiantes situaciones de estrés, angustia, ansiedad y baja autoestima y que, al final, los llevan a desertar de la institución.

En las prácticas pedagógicas de los docentes, sea por vía del trabajo colaborativo para interdisciplinariamente dar respuestas localizadas en esos entornos sociales, sea por atención psicológica o de orientación adecuada, se deben buscar las soluciones creando condiciones en los ambientes relacionales escolares.

Sobre el particular, Beca (2014) se refiere a la necesidad de que los profesores mejoren su experiencia y estudio de los contextos.

Concretamente, el autor afirma que

El estado del arte señala además la necesidad de mejorar la preparación de los profesores para lograr aprendizajes de grupos sociales desfavorecidos, dado que la formación docente es más bien "universalista". También existe una tensión entre la lógica del conocimiento teórico y el dominio de las herramientas pedagógicas prácticas necesarias para la buena enseñanza. (Beca, 2014: 1-2)

De lo dicho hasta aquí se desprende que las variables relacionadas con la deserción escolar varían de acuerdo con los contextos de análisis. Los problemas socioeconómicos y culturales son diferentes según la incidencia de factores tales como niveles de pobreza y pobreza crítica, áreas socioculturalmente deprimidas, niveles 
de escolaridad familiar, violencia intrafamiliar, delictiva en las comunidades y escolar (bulling), carencia de atención psicopedagógica, falta de aplicación de didácticas especiales y escaso interés de los municipios y departamentos en el problema de la pérdida de escolaridad.

La detección oportuna de los motivos de inasistencias permite visualizar, intervenir y detener factores de riesgo que pueden derivar en deserción escolar. En este sentido, es preciso apoyar desde adentro de la escuela al estudiante con problemas familiares y conductuales, lo cual supone un reto a superar desde los conceptos de la teoría sobre la pedagogía del oprimido. Ello no se puede lograr sin crear equipos de trabajo interdisciplinario dispuestos a diagnosticar las falencias que originan la deserción escolar y crear, mediante la mejora y la intervención profesional, las condiciones pedagógicas, sociales, culturales y políticas que remonten las causas de los problemas detectados.

Desde este punto de vista, la deserción es alimentada por factores asociados a la escuela. Las carencias afectivas deben atenderse por programas efectivos en ella. Si no se detectan a tiempo, ni los esquemas pedagógicos y didácticos evalúan esas circunstancias, dichas carencias reforzarán las tendencias a la deserción.

La teoría del trabajo colaborativo aporta mucho sobre este aspecto, que se concreta con la experiencia japonesa y de la Unión Europea al otorgar créditos escolares a profesores que realizan trabajos en equipo para resolver situaciones detectadas en las aulas de clase o individuales. Estos casos se proponen como problemas que deben ser resueltos con propuestas interdisciplinarias a las cuales se les hace seguimiento y evaluación de resultados.

La escuela tradicional repetitiva y memorística no ofrece, en cambio, estrategias para detectar potencialidades, intereses y problemas que permitan una educación más individualizada y propicia a construir conocimientos, recurrir a las experiencias significativas y a posibilitar el "aprender a aprender" como vía de alcanzar los hábitos y herramientas de trabajo individual y práctico que cada quien pueda desarrollar mejor para avanzar escolarmente con sentidos autónomo y crítico.

Es a lo que se refiere Lacueva (2015: 9) cuando señala que lo tradicional de la escuela es una tradición de uniformidad, simplicidad, disciplina impuesta sin más y prácticas pedagógicas rudimentarias: recitaciones, ejercicios, copias, Esta tradición, en buena parte presente aún hoy día, es incapaz de atender a los retos de una preparación más profunda y significativa de las personas.

En general, se puede señalar que con esas características es difícil obtener interés por permanecer en la escuela en aquellos estudiantes susceptibles de desertar.

Los escenarios de organización de las clases y del trabajo escolar deben referenciar el entorno sociocultural, los problemas vivenciales y la manera de trascenderlos con creatividad. De este modo, se superarían las prácticas pedagógicas librescas, rutinarias, reproductoras y alejadas de intereses y expectativas del que aprende Villamizar G. A., Lozano, S. G., y Sierra, E. D. (2017).

$\mathrm{Si}$ se crean condiciones para aprender a aprender, en las cuales la autonomía del estudiante se haga presente responsablemente, y el profesor realice interacciones entre el saber académico, la conciencia ordinaria y la actividad crítica (sustentadas en realidades que aborden las experiencias de vida de los estudiantes y de cómo superar los problemas en ellas existentes), se estará contribuyendo a crear condiciones emocionales e intelectuales para potenciar la continuidad de la escolaridad e identificar los casos que requieren de los apoyos profesionales, de servicios sociales y psicológicos accesibles en la institución escolar.

\section{El problema de la deserción escolar en Colombia}

En nuestro país, desde las políticas públicas y documentos que las sustentan, se aborda la problemática de la deserción. Así, por ejemplo, en el documento "Acuerdo por lo superior", se habla de la innovación social para atender los problemas 
generados por la pobreza, la crisis social y la política del país. Ya aparece en la agenda del gobierno atender estos asuntos desde la "Agencia Nacional para Superación de la Pobreza Extrema, ANSPE, y el Centro de Innovación Social, CIS"; para que "Colombia en los próximo 20 años supere estos problemas a partir de una política pública" (MEN, 2014: 102).

Las propuestas de inclusión escolar sugeridas por la UNESCO y contempladas en los documentos de las políticas públicas en Colombia, insisten en modificar las condiciones socioeconómicas que conllevan a la deserción escolar con el objetivo, de acuerdo con las realidades locales y regionales, de disminuir progresivamente este fenómeno que afecta la calidad de la educación en general y las posibilidades de un desarrollo sostenible con equidad.

La deserción entonces, consiste en la pérdida paulatina de los efectivos escolares en un sistema educativo estimulada por esos factores. En Colombia la deserción alcanza hasta más del 40\% entre la etapa primaria y secundaria (MEN, 2014), y los factores que inciden en ella se deben abordar con políticas públicas que atiendan los problemas socioeconómicos en las comunidades marginales y vulnerables. Pero también se requiere del factor pedagógico que eleve la motivación, participación e interés de los estudiantes hacia las expectativas de valor y cambio humano que permiten la educación.

Moreno (2013) señala que en Colombia se trabaja en el cumplimiento de las políticas educativas destinadas a hacer cumplir la norma constitucional del artículo 67 que establece la educación obligatoria entre los cinco y los quince años de edad, y si las metas propuestas sobre cobertura educativa se alcanzan a niveles nacional, departamental y municipal; así como la importancia otorgada a que los "estudiantes desarrollen sus competencias básicas y ciudadanas con herramientas para la construcción de sus proyectos de vida" (Moreno, 2013: 115-116).

También el trabajo abarca la importancia de la lectura comprensiva, competencia básica a estimularse en Colombia desde la educación formal. Los resultados de las últimas pruebas PISA en las que el país ha participado muestran falencias en esa área. La superación de estas limitaciones se convertiría en factor que contrarreste el desapego a la escuela, puesto que la lectura aviva el interés en los alumnos por aprender y ampliar la mirada al origen del problema de deserción escolar.

El Ministerio de Educación Nacional en Colombia invirtió para lograr mayor cobertura de educación y así cumplir con las políticas públicas referidas a transformar a Colombia en el país más educado de América Latina para el 2025; sin embargo, tales políticas parecen no estar disminuyendo sustancialmente la deserción escolar.

Son múltiples las causas por las que el estudiante deserta de la educación formal, aunque el Ministerio de Educación Nacional, por medio de las instituciones escolares trata de mitigarlas. Por ejemplo, cuando se habla de la causa monetaria, el gobierno colombiano, desde el año 2003,estableció la educación básica y secundaria gratuita para estudiantes de estratos 1 y 2, y para el año 2008 se instituyó la educación gratuita hasta bachiller medio. Aparte, algunas instituciones educativas apoyan a la comunidad brindando subsidios, o el mismo Estado también lo hace con el fin de ayudar a estudiantes de escasos recursos para que puedan asistir a la escuela. (MEN, 2012).

Otra de las causas de la deserción es la mala conducta del estudiante en el plantel educativo, ante lo cual las entidades escolares tienden a extender

\footnotetext{
${ }^{1}$ Constitución Política de Colombia, artículo 67. La educación es un derecho de la persona y un servicio público que tiene una función social; con ella se busca el acceso al conocimiento, a la ciencia, a la técnica, y a los demás bienes y valores de la cultura. La educación formará al colombiano en el respeto a los derechos humanos, a la paz y a la democracia; y en la práctica del trabajo y la recreación, para el mejoramiento cultural, científico, tecnológico y para la protección del ambiente. El Estado, la sociedad y la familia son responsables de la educación, que será obligatoria entre los cinco y los quince años de edad y que comprenderá como mínimo, un año de preescolar y nueve de educación básica.
} 
al máximo el apoyo al estudiante para que mejore su comportamiento a fin de evitar su expulsión. Por ley, la Constitución Política de Colombia, en el artículo 67, reconoce la importancia del derecho de la educación. También el artículo 96 de la Ley 115 de 1994 regula la permanencia del estudiante dentro del plantel educativo. No obstante, cuando el estudiante mantiene la actitud de indisciplina, el plantel recomienda cambiarlo de institución para que pueda continuar con sus estudios, aunque la decisión final es del estudiante y su acudiente.

De acuerdo con el Departamento Administrativo Nacional de Estadística (DANE), las cifras de deserción en Colombia son preocupantes. Según estudios de esta dependencia, la deserción escolar disminuyó escasamente el $0,4 \%$ entre 2011 y 2012. Otro estudio (García Jaramillo, Fernández Monsalve y Sánchez Torres, 2010), mostró que de 10.641.243 estudiantes matriculados en el año 2012, la cantidad se redujo a 10.540.711; en el año 2013, entre quienes cursaban preescolar y básica primaria la caída fue de $1,3 \%$, y de secundaria básica y media descendió $0,5 \%$.

Sobre la base de la información suministrada por las instituciones escolares, el Ministerio de Educación Nacional lleva un seguimiento estadístico para conocer la cantidad de estudiantes que se matriculan año a año con el fin de evitar la deserción escolar. (MEN, 2009).

Teniendo presente que los factores que inciden con mayor fuerza en el abandono estudiantil son el económico, el cultural y el socio-afectivo de origen familiar, el plantel educativo, como interventor directo con el estudiante, es quien muchas veces debe crear estrategias de solución, pues la intervención de los padres en la educación de sus hijos y la aplicación de estrategias pedagógicas pertinentes y agradables, también es válida para intervenir sobre estos dos fenómenos de ineficiencia escolar.

Generalmente los planes regionales contemplan apoyos para atender este problema de la deserción y alcanzar un mayor desarrollo humano (Gobernación de Norte de Santander, 2011) y a través de la investigación lograr "solucionar problemas de la región”, para lo cual se hace necesario llegar a acuerdos entre autoridades regionales y nacionales de manera colaborativa, pues la educación es tomada como "foco estratégico... por ser uno de los pilares del plan nacional de desarrollo 2016-2020". (Gobernación de Norte de Santander, 2015).

\section{Recursos materiales y métodos que deben tenerse en cuenta para la investigación sobre deserción escolar}

\subsection{Posicionamiento teórico}

La metodología elegida para la investigación sobre temas de deserción escolar debe estar cercana al objeto de estudio a fin de dar explicaciones y recomendaciones pertinentes. La interacción entre sujeto y objeto requiere continuidad en el estudio de este fenómeno, pues fortalece la reflexión sobre los procesos que inciden en él y otorga confiabilidad al contenido crítico de carácter cualitativo.

Necesario es indagar esa realidad con los propios sujetos comprometidos frente a la institución escolar como es el caso de directivos, profesores, acudientes y alumnos para obtener la información suficiente y necesaria que permita soluciones atinentes al problema. Por tanto,

El método debe ser preferiblemente abierto, propicio a revisar procesos complejos, globalizadores, flexibles y cambiantes, utilizando técnicas e instrumentos básicamente no estructurados, que permitan obtener resultados a partir de la acción consciente del investigador, no generalizables, que potencien propuestas científicas. (Hernández, 2002: 55).

La etnografía nos permite esta caracterización por cuanto procura la ubicación en un contexto geográfico y social determinado en el cual sus protagonistas producen información para el diagnóstico del problema y su posible solución. Además, tal y como sostiene Martínez (1998: 2930), 
la unidad de análisis para el investigador, no solo podría ser una nación, un grupo lingüístico, una región o una comunidad, sino también cualquier grupo humano que constituya una identidad cuya relaciones estén reguladas por la costumbre o por ciertos derechos y obligaciones recíprocos. Así como, en la sociedad moderna una familia, una institución educativa, un aula de clase, una fábrica, una empresa, un hospital, una cárcel un gremio obrero, un club social etc.., son unidades sociales que pueden ser estudiadas etnográficamente.

Dada la necesidad de evitar deserción escolar convenientes que el fenómeno se estudie desde la perspectiva etnográfica. No todas las instituciones y escenarios socioculturales, son iguales y, por tanto, requieren de procesos metodológicos diferenciados a objeto de comprender mejor las particularidades del contexto, observar las causas que generan los problemas y las diversas formas de abordarlos.

Se debe dar un carácter interpretativo a la forma de estudiar el problema. Por tanto, la hermenéutica será clave en todo el proceso para entender cómo intervienen los factores de análisis en el resultado que permita diagnosticar las causas de la deserción en la institución, para lo cual se debe triangular la información proveniente de los sujetos y fuentes de información.

Es conveniente escoger el muestreo de conveniencia intencional como forma metodológica de implementar en este estudio. Por tanto, será suficiente analizar el problema en instituciones escolares de sectores vulnerables, pues son más representativas de las dificultades sociales, económicas y familiares que inciden en el abandono de la escuela.

A continuación se presenta un diseño que representa desde el punto de vista metodológico el problema en cuestión:

Figura 1

Diseño de la investigación

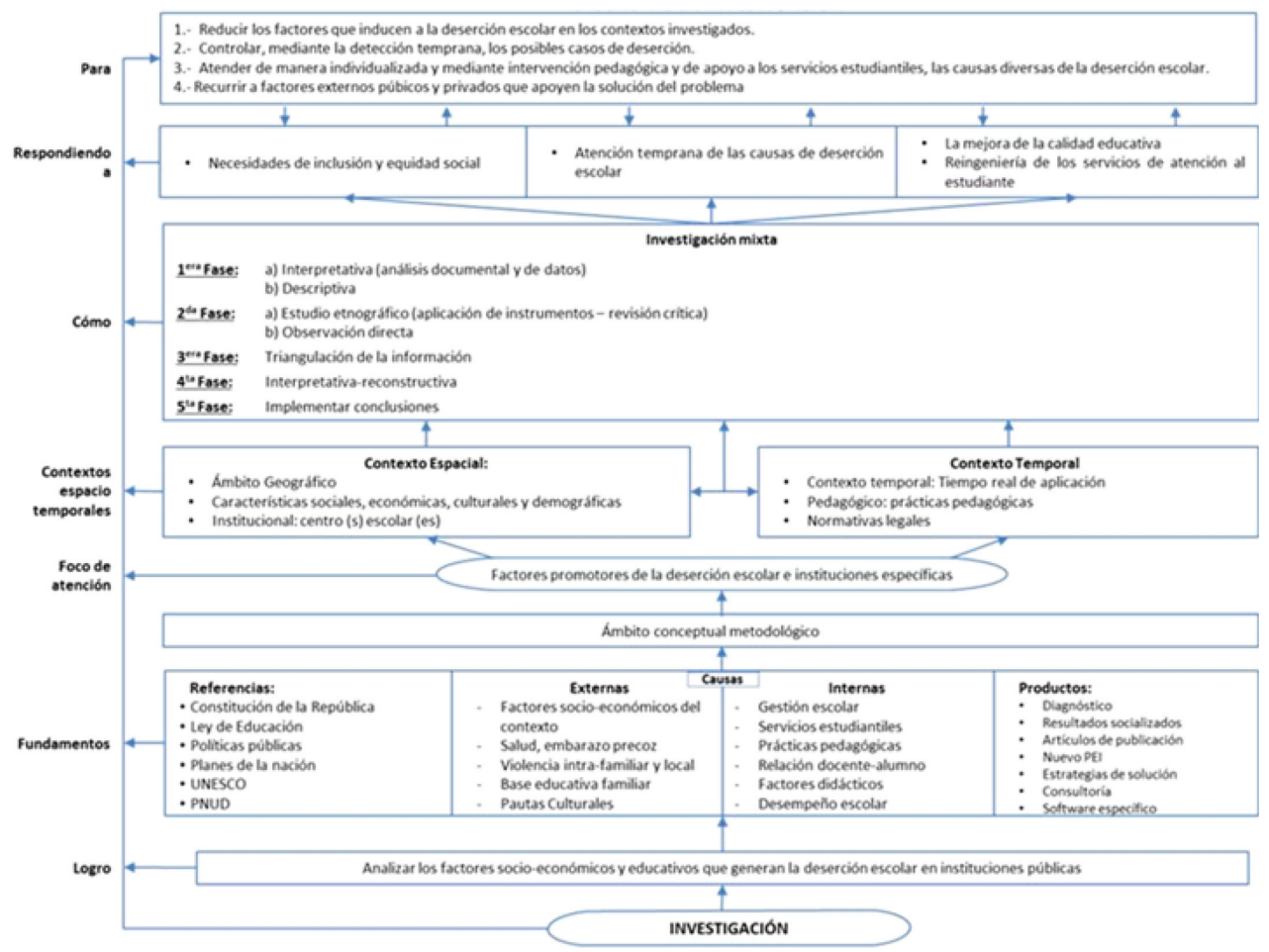

Fuente: Hernández Dávila y Díaz Abdala. Proceso de investigación. 


\subsection{Establecimiento de las poblaciones $y$ muestras en las investigaciones sobre deserción}

Estudiantes, directivos, profesores, padres y representantes que se encuentran en instituciones de contextos vulnerables. Pueden escogerse instituciones públicas, de zonas urbanas y rurales, de alta matrícula, que permitan la participación en el proceso investigativo. La población debe seleccionarse de acuerdo con los juicios de inclusión y exclusión, partiendo del número de menores que han desertado durante los últimos períodos escolares.

\subsection{Recolección de la información}

La información se iniciará con análisis de documentos que presenten las estadísticas de ingreso y culminación en los últimos años. Los observadores harán revisión de las historias de vida de alumnos con conductas irregulares, recibiendo la opinión de orientadores.

Se recomienda aplicar encuesta y entrevistas a directivos, estudiantes, profesores y padres de familias o acudientes, con preguntas alusivas al fenómeno de la deserción escolar, cuyas variables principales deben extraerse de los contextos de análisis. Igualmente, mediante observación directa, se registrará información sobre el estado de la planta física, instalaciones deportivas, aulas de clase, laboratorios, áreas de recreación y las destinadas a materiales didácticos.

\subsection{Criterios de inclusión}

Menores de edad que al momento de realizar la encuesta sus padres deseen participar en la investigación.

\subsection{Criterios de exclusión}

Menores de edad cuyos padres no deseen participar en la investigación al momento de realizar la encuesta.

\subsection{Necesidad del consentimiento informado}

Socializar en detalle las condiciones, beneficios y riesgos del proceso en un lenguaje sencillo para que las personas puedan ejercer su libre albedrío. Esto a fin de solicitar la autorización a los participantes del estudio para que suministren información y esta pueda ser utilizado en la investigación. Se tendrán en cuenta las siguientes consideraciones: a) Notificación a la persona sobre los procedimientos que se van a realizar, riesgos e incomodidades, así como sus derechos. b) Declaración de que la participación es voluntaria y que la negativa a participar no implica represalias, castigos o pérdida de algún beneficio. c) Descripción de los beneficios que se espera obtener. d) Descripción de procedimientos alternativos. e) Compromiso del investigador responsable sobre la confidencialidad de los datos. f) Descripción del riesgo e información acerca de éste. g) Firma de las partes (investigador y participante).

\subsection{Consideraciones éticas}

Responsabilidad de los investigadores integrantes del proyecto ante el compromiso de desarrollar una investigación mixta conforme a las normativas institucionales vigentes y a los tiempos establecidos para concluirla.

Esta investigación requiere obtener información válida y confiable de documentos pertenecientes a la institución objeto de estudio, de padres, profesores y alumnos; en consecuencia, es imprescindible, garantizar la confiabilidad de las fuentes.

Se seguirán las recomendaciones en aspectos éticos para la investigación en seres humanos como el Código de Nuremberg, Declaración de Helsinki, y el estatuto de Roma, entre otros.

En conclusión, se debe respetar la decisión de cada sujeto de participar o no en la investigación, no perjudicar a algún participante en el estudio, garantizar el secreto de información de las fuentes y que los beneficios derivados de la investigación se repartan de forma razonables socialmente. 


\section{Orientaciones para el análisis de resultados}

La credibilidad en la investigación se obtendrá de los procesos de triangulación de la información obtenida de estudiantes, profesores, directivos y padres de familias o acudientes. Toda esa variedad de fuentes se contrastará para elaborar el análisis ajustado al carácter cualitativo de la investigación.

La variedad de fuentes estará sujeta a interpretaciones, sustento base de la investigación cualitativa, aunada al análisis de la normativa legal, a los referentes teóricos y documentales consultados; dando lugar al legado conceptual, creíble y fiable que el estudio debe contener.

También los objetivos de la investigación serán guías del trabajo para apreciar si ella se dirige por buen camino y si estos se lograron al finalizar; permitiendo elaborar las estrategias de retención y permanencia, la aplicación de las recomendaciones del caso y el apoyo de las instituciones a las soluciones pertinentes de la problemática detectada.

\subsection{Resultados esperados}

Se deben concebir resultados con efectos de transferibilidad, porque el objeto de estudio (la deserción escolar) tiene múltiples determinaciones, que pese a ser particulares e irrepetibles, contribuyen a proponer intervenciones positivas en realidades similares y coincidentes, sobre todo si se aplican en aquellos circuitos de carencias donde el fenómeno tiende a agudizarse.

Al finalizarla investigación se apreciarán las condiciones externas e internas que posiblemente influyen en la deserción escolar de los menores. Asimismo, la información obtenida servirá de referente a estrategias que desde las políticas públicas surjan en los ámbitos educativo y social.

\subsection{Productos esperados}

Respetando el análisis realizado en este trabajo, se debe buscar garantizar la obtención de los siguientes productos de investigación: a)
Producir un diagnóstico sobre el problema de la deserción en la (s) institución (es) escogida (s) como objeto de estudio. b) Los aportes surgidos como nuevo conocimiento deben hacerse conocer en la comunidad educativa para empoderarla y que participe en la solución del problema. c) Elaborar un artículo académico con los resultados del trabajo donde se resalte los aportes innovadores de la experiencia. d) Incorporar al Proyecto Educativo Institucional (PEI) en su visión, gestión y objetivos los resultados de investigaciones de esta naturaleza, para fortalecer la calidad educativa con intervenciones e innovaciones adecuadas a los nuevos criterios de control que requiere la deserción escolar. e) A objeto de que exista apropiación social del conocimiento, difundir los resultados de la investigación a entes gubernamentales, nacionales y regionales, que puedan generar políticas públicas. f) Servir de fuente de análisis en los trabajos en equipos colaborativos que surjan en las instituciones escolares objeto de estudio y así se fortalezcan la aplicación de criterios de formación permanente de los profesores, basados en el estudio de las prácticas pedagógicas e investigación del currículo. g) De los estudios específicos, en conformidad con el contexto de análisis, se pueden obtener los datos e información necesarios para, con la ayuda de expertos en TIC's, se construyan software responsivos a las necesidades particulares de las comunidades educativas donde el fenómeno se investigue.

\section{Bibliografía}

Abril Valdez, E.; Román Pérez, R.; Cubillas Rodríguez, M.J.; y Moreno Celaya, I. (2008). ¿Deserción o autoexclusión? Un análisis de las causas de abandono escolar en estudiantes de educación media superior en Sonora, México. Revista electrónica de investigación educativa, 10(1), 1-16. Disponible en http://www.scielo.org. $\mathrm{mx} /$ scielo.php?script $=$ sci_arttext\&pid $=\mathrm{S} 1607$ 40412008000100007\&lng=es\&tlng=es.

Beca, C.E. (2014). Estrategia regional docente. Conferencia presentada en el VI Congreso 
Iberoamericano de Pedagogía. Valparaíso: Pontificia Universidad Católica de Valparaíso, 23 y 24 de septiembre. Disponible en http:// www.congresodepedagogia.ucsh.cl

Consejo Nacional de Educación Superior. (2014). Acuerdo por lo superior. Disponible en http:// www.dialogoeducacionsuperior.edu.co/1750/ articles-319917_recurso_1.pdf

Dirección Administrativa Nacional de Estadística. (2016)Investigación de Educación Formal 2016. Disponible en http://www.dane.gov.co/ index.php/estadisticas-por-tema/educacion

El Espectador. (2016). Preocupantes cifras de deserción escolar. [En línea] 25 de septiembre de 2015]. Disponible en http://www.elespectador. com/noticias/educacion/preocupantes-cifras-dedesercion-escolar-articulo-518837.

Esteban, R. (2016). Tendencias pedagógicas. Educación y educación superior en el contexto iberoamericano. Madrid: Universidad Autónoma de Madrid.

García Jaramillo, S.; Fernández Monsalve, C. y Sánchez Torres, F. (2010). Deserción y repetición en los primeros grados de la básica primaria: factores de riesgo y alternativas de política pública. Disponible en http:// www.educacioncompromisodetodos.org/ datos/274A1_ECT\%20Desercion\%20y\%20 repeticion.pdf

Gobernación de Norte de Santander. (2011) Plan de Desarrollo para Norte de Santander 2012-2015. Disponible en https://ids.gov.co/ web/planesids/plan-de-desarrollo-norte-desantander-2012-2015.pdf

Gobernación de Norte de Santander. (2015) Plan de Desarrollo del Departamento de Norte de Santander 2016-2019. Disponible en http:// www.siipe.co/wp-content/uploads/2014/08/ Plan-Norte-de-Santander.pdf

Hernández, Román. (2002). La formación permanente del profesorado desde los centros educativos. Mérida: Universidad de los Andes.

La cueva, A. (2015). La investigación en la escuela. Proyecto y actividades y recursos. Caracas:
Editorial Casa de la Cultura.

Martínez, M. (1998). La investigación cualitativa etnográfica en educación. Manual teórico práctico. México D.F.: Trillas.

Ministerio de Educación Nacional. (2009). Educación básica y media. Disponible en http://www.mineducacion.gov.co/1621/ article-188917.html.

Ministerio de Educación Nacional.(2012). La Secretaria de Educación del Distrito Capital entrega 10 mil nuevos subsidios educativos a estudiantes de Bogotá. Disponible en http://www. mineducacion.gov.co/cvn/1665/article-188458. html.

Moreno D. (2013). La deserción escolar: Un problema de carácter social. In Vestigium Ire, 6: 115-116.

National Dropout Prevention Center. (2017). Our Mission. Disponible en http://dropoutprevention. org/who-we-are/our-mission/

Spindola, E. y León, A. (2002). La deserción escolar en América Latina: un tema prioritario para la agenda regional. Revista Iberoamericana de Educación, 30: 39-62. Disponible en http:// www.seg.guanajuato.gob.mx/Ceducativa/ CartillaB/6antologia/antecedentes/ pdf/32.20LA \%20DESERCI\%C3\%93N\%20 ESCOLAR\%20EN\%20AM\%C3\%89RICA\%20 L A T I N A \% 20 U N \% 20 T E M A \% 20 PRIORITARIO \% 20 PARA \% 20 LA \% 20 AGENDA\%20REGIONAL.pdf

UNESCO. (2013). Situación Educativa de América Latina y el Caribe: Hacia la educación de calidad para todos 2015. Santiago de Chile: UNESCO.

UNESCO. (2013). Situación educativa de América Latina y el Caribe: Hacia la educación de calidad para todos al 2015. Santiago de Chile: Oficina Regional de Educación para América Latina y el Caribe (OREALC/UNESCO Santiago). Disponible en ttp://www.unesco.org/new/ fileadmin/MULTIMEDIA/FIELD/Santiago/ images/SITIED-espanol.pdf

Villamizar G. A., Lozano, S. G., y Sierra, E. D. (2017). El Conocimiento desde la Perspectiva del Estudiante. Perspectivas. 2 (1). 18-7 